\title{
A generalization of very odd sequences
}

\author{
Cheng Yeaw $\mathrm{Ku}$ \\ Department of Mathematics \\ National University of Singapore \\ Singapore 117543. \\ matkcy@nus.edu.sg
}

\author{
Kok Bin Wong \\ Institute of Mathematical Sciences \\ University of Malaya \\ 50603 Kuala Lumpur, Malaysia \\ kbwong@um.edu.my
}

Submitted: Mar 2, 2015; Accepted: Apr 6, 2015; Published: Apr 21, 2015

Mathematics Subject Classifications: 11B50, 11B83

\begin{abstract}
Let $\mathbb{N}$ be the set of positive integers and $n \in \mathbb{N}$. Let $\mathbf{a}=\left(a_{0}, a_{1}, \ldots, a_{n-1}\right)$ be a sequence of length $n$, with $a_{i} \in\{0,1\}$. For $0 \leqslant k \leqslant n-1$, let

$$
A_{k}(\mathbf{a})=\sum_{\substack{0 \leqslant i \leqslant j \leqslant n-1 \\ j-i=k}} a_{i} a_{j} .
$$

The sequence $\mathbf{a}$ is called a very odd sequence if $A_{k}(\mathbf{a})$ is odd for all $0 \leqslant k \leqslant$ $n-1$. In this paper, we study a generalization of very odd sequences and give a characterisation of these sequences.
\end{abstract}

Keywords: very odd sequence, Pelikán's conjecture

\section{Introduction}

Let $\mathbb{N}$ be the set of positive integers and $n \in \mathbb{N}$. Let $\mathbf{a}=\left(a_{0}, a_{1}, \ldots, a_{n-1}\right)$ be a sequence of length $n$, with $a_{i} \in\{0,1\}$. For $0 \leqslant k \leqslant n-1$, let

$$
A_{k}(\mathbf{a})=\sum_{\substack{0 \leqslant i \leqslant j \leqslant n-1 \\ j-i=k}} a_{i} a_{j}
$$

The sequence $\mathbf{a}$ is called a very odd sequence if $A_{k}(\mathbf{a})$ is odd for all $0 \leqslant k \leqslant n-1$.

Pelikán [6] conjectured that very odd sequences of length $n \geqslant 5$ do not exist. Later, Alles [1] and MacWilliams and Odlyzko [4] proved that Pelikán conjecture is false (see also [5]). In fact, Inglis and Wiseman [2] and MacWilliams and Odlyzko [4] proved the following theorem which gives a necessary and sufficient condition for the existence of a very odd sequences of length $n$. 
Theorem 1. A very odd sequence of length $n>1$ exists if and only if the order of 2 is odd in the multiplicative group of integers modulo $2 n-1$.

Let $p$ be a prime and $\mathbf{z}=\left(z_{0}, z_{1}, z_{2}, \ldots\right)$ be an infinite sequence. A sequence $\mathbf{a}=$ $\left(a_{0}, a_{1}, \ldots, a_{n-1}\right)$ of length $n$ with $a_{i} \in \mathbb{N} \cup\{0\}$ is called a $(\mathbf{z}, p)$-sequence if

$$
A_{k}(\mathbf{a}) \equiv z_{k} \quad \bmod p, \quad \forall 0 \leqslant k \leqslant n-1 .
$$

For each $k \in \mathbb{N} \cup\{0\}$, let $\bar{k}=(k, k, k, \ldots)$ be the infinite sequence with all entries equal to $k$. Then, Theorem 1 can be rewritten as follows:

Theorem 2. $A(\overline{1}, 2)$-sequence of length $n>1$ exists if and only if the order of 2 is odd in the multiplicative group of integers modulo $2 n-1$.

In this paper, we give necessary and sufficient conditions for the existence of a $(\bar{k}, p)$ sequence of length $n>1$ (Theorem 12). We will also consider the existence of a $\left(\mathbf{y}_{k}, p\right)$ sequence of length $n>1$ (Theorem 14) where $\mathbf{y}_{k}=\left(y_{0}, y_{1}, y_{2}, \ldots\right)$ and $y_{i}=(-1)^{i} k$.

\section{Main Results}

Let $p$ be a prime and $\mathbb{Z}_{p}$ be the field with $p$ elements. We shall denote the set of all polynomials over the field $\mathbb{Z}_{p}$ by $\mathbb{Z}_{p}[x]$. For any sequence $\mathbf{a}=\left(a_{0}, a_{1}, \ldots, a_{n-1}\right)$ of length $n$ with $a_{i} \in \mathbb{N} \cup\{0\}$, we set

$$
f_{\mathbf{a}}(x)=a_{0}+a_{1} x+\cdots+a_{n-1} x^{n-1} .
$$

Then $f_{\mathbf{a}}(x) \in \mathbb{Z}_{p}[x]$.

For a polynomial $g(x)=c_{0}+c_{1} x+\cdots+c_{n-1} x^{n-1} \in \mathbb{Z}_{p}[x]$, we set

$$
g^{*}(x)=c_{n-1}+c_{n-2} x+\cdots+c_{0} x^{n-1} .
$$

Note that $g^{*}(x)=x^{n-1} g\left(\frac{1}{x}\right)$ and $\left(g^{*}(x)\right)^{*}=g(x)$. Furthermore, $f_{\mathbf{a}}^{*}(x)=f_{\mathbf{a}^{*}}(x)$ where $\mathbf{a}^{*}=\left(a_{n-1}, \ldots, a_{1}, a_{0}\right)$ is the reverse of $\mathbf{a}=\left(a_{0}, a_{1}, \ldots, a_{n-1}\right)$.

The following two lemmas are obvious.

Lemma 3. Let $f(x), g(x), h(x)$ be polynomials of degree at least 1 in $\mathbb{Z}_{p}[x]$. If $f(x)=$ $g(x) h(x)$, then $f^{*}(x)=g^{*}(x) h^{*}(x)$.

Lemma 4. If $f(x)$ is a monic irreducible polynomial in $\mathbb{Z}_{p}[x]$, then $\frac{1}{f(0)} f^{*}(x)$ is also a monic irreducible polynomial in $\mathbb{Z}_{p}[x]$.

Lemma 5. A sequence $\mathbf{a}=\left(a_{0}, a_{1}, \ldots, a_{n-1}\right)$ is a $(\mathbf{z}, p)$-sequence if and only if

$$
f_{\mathbf{a}}(x) f_{\mathbf{a}}^{*}(x)=\sum_{i=0}^{n-1} z_{n-1-i} x^{i}+x^{n-1} \sum_{i=1}^{n-1} z_{i} x^{i},
$$

in $\mathbb{Z}_{p}[x]$. 
Proof. Note that

$$
\begin{aligned}
f_{\mathbf{a}}(x) f_{\mathbf{a}}^{*}(x) & =\left(\sum_{i=0}^{n-1} a_{i} x^{i}\right)\left(\sum_{j=0}^{n-1} a_{j} x^{n-1-j}\right) \\
& =\sum_{l=0}^{2 n-2}\left(\sum_{\substack{0 \leqslant i, j \leqslant n-1 \\
j-i=n-1-l}} a_{i} a_{j}\right) x^{l} \\
& =\sum_{l=0}^{2 n-2} A_{|n-1-l|}(\mathbf{a}) x^{l} \\
& =\sum_{i=0}^{n-1} A_{n-1-i}(\mathbf{a}) x^{i}+x^{n-1} \sum_{i=1}^{n-1} A_{i}(\mathbf{a}) x^{i} .
\end{aligned}
$$

The lemma follows by noting that $\mathbf{a}=\left(a_{0}, a_{1}, \ldots, a_{n-1}\right)$ is a $(\mathbf{z}, p)$-sequence if and only if $A_{i}(\mathbf{a}) \equiv z_{i} \bmod p$ for all $i$.

The following corollary follows immediately from Lemma 5.

Corollary 6. If $k \equiv 0 \bmod p$, then there is exactly one $(\bar{k}, p)$-sequence of length $n>1$, which is $(\overbrace{0,0, \ldots, 0}^{n})$.

We shall require the following lemmas.

Lemma 7. ([3, Theorem 2.14 on p. 128]) Let $f(x)$ and $g(x) \neq 0$ be polynomials in $F[x]$, where $F$ is a field. Then there exist polynomials $q(x)$ and $r(x) \in F[x]$ with the degree of $r(x)$ less than the degree of $g(x)$ such that $f(x)=q(x) g(x)+r(x)$.

Lemma 8. ([3, Theorem 4.26 on p. 288]) Let $F$ be a finite field with $q=p^{m}$ elements and $E$ be a field extension of $F$ with $[E: F]=n$. Then the Galois group $G(E / F)$ is a cyclic group with generator $\eta$, where $\eta: a \rightarrow a^{q}$.

Note that the Galois group $G(E / F)$ is the group of all automorphisms of $E$ that fix $F$, i.e., $\theta \in G(E / F)$ if and only if $\theta(a)=a$ for all $a \in F$ and $\theta \in \operatorname{Aut}(E)$ the group of all automorphisms of $E$.

Lemma 9. ([3, Section 4.4 on p. 229]) Let $f(x) \in \mathbb{Z}_{p}[x]$ and $f^{\prime}(x)$ be the formal derivative of $f(x)$. If $\beta$ is a multiple root of $f(x)$, then $f^{\prime}(\beta)=0$.

We denote the greatest common divisor of $c, d$ by $\operatorname{gcd}(c, d)$.

Lemma 10. Let $\operatorname{gcd}(p, 2 n-1)=1=\operatorname{gcd}(p-1,2 n-1)$ and $\beta$ be a root of $\sum_{i=0}^{2 n-2} x^{i}$. If $h(x) \in \mathbb{Z}_{p}[x]$ is a monic irreducible polynomial with $h(\beta)=0$ and the order of $p$ modulo $2 n-1$ is odd, then the degree of $h(x)$ is odd and $h(0)=-1$. Furthermore, $-h^{*}(x) \neq h(x)$. 
Proof. Let $E$ be a field extension of $\mathbb{Z}_{p}$ containing $\beta$. Let the order of $\beta$ in $E$ be $t$, i.e., $t$ is the least positive integer such that $\beta^{t}=1$. Note that $(x-1) \sum_{i=0}^{2 n-2} x^{i}=x^{2 n-1}-1$. So, $\beta$ is a root of $x^{2 n-1}-1$, i.e., $\beta^{2 n-1}=1$. This implies that $t$ divides $2 n-1$ and $\operatorname{gcd}(p, t)=1=\operatorname{gcd}(p-1, t)$. Let the order of $p$ modulo $t$ be $e$. Then $\beta^{p^{e}}=\beta$ and $\beta^{p^{i}} \neq \beta$ for $1 \leqslant i \leqslant e-1$. Furthermore, $e$ is odd as the order of $p$ modulo $2 n-1$ is odd.

By Lemma 8 , the Galois group $G\left(E / \mathbb{Z}_{p}\right)$ is a cyclic group with generator $\eta$. Note that $\eta\left((x-\beta)\left(x-\beta^{p}\right) \ldots\left(x-\beta^{p^{e-1}}\right)\right)=(x-\beta)\left(x-\beta^{p}\right) \ldots\left(x-\beta^{p^{e-1}}\right)$. So, $(x-\beta)(x-$ $\left.\beta^{p}\right) \ldots\left(x-\beta^{p^{e-1}}\right) \in \mathbb{Z}_{p}[x]$ and $h(x)=(x-\beta)\left(x-\beta^{p}\right) \ldots\left(x-\beta^{p^{e-1}}\right)$. Thus, the degree of $h(x)$ is $e$ which is odd.

Now, $(p-1)\left(1+p+\cdots+p^{e-1}\right)=p^{e}-1 \equiv 0 \bmod t$. Since $\operatorname{gcd}(p-1, t)=1$, we have $1+p+\cdots+p^{e-1} \equiv 0 \bmod t$. Therefore $h(0)=(-1)^{e} \beta^{1+p+\cdots+p^{e-1}}=(-1)^{e}=-1$.

By Lemma $4,-h^{*}(x)$ is a monic irreducible polynomial and $-h^{*}\left(\beta^{-1}\right)=0$. Suppose $-h^{*}(x)=h(x)$. Then $\beta^{p^{i_{0}}}=\beta^{-1}$ for some $0 \leqslant i_{0} \leqslant e-1$. This implies that $p^{i_{0}} \equiv-1$ $\bmod t$ and $p^{2 i_{0}} \equiv 1 \bmod t$. So, $e$ divides $2 i_{0}$, and $e$ divides $i_{0}$ for $e$ is odd. This means that $p^{i_{0}} \equiv 1 \bmod t$ and $2 \equiv 0 \bmod t$. Therefore, $t=1$ or 2 . If $t=1$, then $\beta=1$ and $0=\sum_{i=0}^{2 n-2} \beta^{i}=2 n-1\left(\right.$ in $\left.\mathbb{Z}_{p}\right)$, contradicting the fact that $\operatorname{gcd}(p, 2 n-1)=1$. If $t=2$, then 2 divides $2 n-1$, which is another contradiction. Hence, $-h^{*}(x) \neq h(x)$.

Lemma 11. Let $F$ be a field. Then $x^{m_{1}}-1=\left(x^{m_{2}}-1\right) w(x)$ for some polynomial $w(x) \in F[x]$ if and only if $m_{2}$ divides $m_{1}$.

Proof. Let $m_{1}=q m_{2}+r$ where $r, q$ are integers with $0 \leqslant r<m_{2}$. Note that

$$
x^{m_{1}}-1=x^{q m_{2}+r}-1=\left(x^{m_{2}}-1\right)\left(x^{(q-1) m_{2}+r}+x^{(q-2) m_{2}+r}+\cdots+x^{m_{2}+r}+x^{r}\right)+x^{r}-1 .
$$

It then follows from Lemma 7 that $x^{m_{1}}-1=\left(x^{m_{2}}-1\right) w(x)$ for some polynomial $w(x) \in$ $F[x]$ if and only if $r=0$.

For each $d \in \mathbb{N}$, let $\mathbb{Z}_{d}$ be the ring of integers modulo $d$ and $U_{d}$ be the multiplicative group of units in $\mathbb{Z}_{d}$.

Theorem 12. Let $p$ be a prime, $k \in \mathbb{Z}_{p} \backslash\{0\}$ and $\operatorname{gcd}(p, 2 n-1)=1=\operatorname{gcd}(p-1,2 n-1)$. $A(\bar{k}, p)$-sequence of length $n>1$ exists if and only if

(a) the order of $p$ is odd in $U_{2 n-1}$,

(b) $(-1)^{n-1} k$ is a quadratic residue modulo $p$.

Furthermore, if such a sequence exists, then there are exactly $2^{l}$ of them if $p=2$ and $2^{l+1}$ if $p$ is odd, where $2 l$ is the number of irreducible factors of $\sum_{i=0}^{2 n-2} x^{i}$.

Proof. $(\Rightarrow)$ Let $\mathbf{a}=\left(a_{0}, a_{1}, \ldots, a_{n-1}\right)$ be a $(\bar{k}, p)$-sequence of length $n>1$. By Lemma 5 ,

$$
f_{\mathbf{a}}(x) f_{\mathbf{a}}^{*}(x)=k \sum_{i=0}^{2 n-2} x^{i} .
$$


Note that $h(x)=(x-1) \sum_{i=0}^{2 n-2} x^{i}=x^{2 n-1}-1$ and $h^{\prime}(x)=(2 n-1) x^{n-2} \neq 0$ in $\mathbb{Z}_{p}[x]$ for $\operatorname{gcd}(p, 2 n-1)=1$. It follows from Lemma 9 that $h(x)$ has no multiple roots. Thus, $\sum_{i=0}^{2 n-2} x^{i}$ has no multiple roots.

Note that $a_{n-1} a_{0}=k \not \equiv 0 \bmod p$ for $a_{n-1} a_{0}$ is the coefficient of $x^{2 n-2}$ in $f_{\mathbf{a}}(x) f_{\mathbf{a}}^{*}(x)$. So, $a_{n-1} \not \equiv 0 \bmod p$. Let $f_{\mathbf{a}}(x)=a_{n-1} q_{1}(x) q_{2}(x) \ldots q_{m}(x)$ where each $q_{i}(x)$ is a monic irreducible polynomial.

Suppose $p$ is of even order in $U_{2 n-1}$. Let $2 l$ be the order of $p$ modulo $2 n-1$. Then $\left(p^{l}-1\right)\left(p^{l}+1\right)=p^{2 l}-1 \equiv 0 \bmod (2 n-1)$. Let $\beta_{1}, \beta_{2}, \ldots, \beta_{2 n-2}$ be all the distinct roots of $\sum_{i=0}^{2 n-2} x^{i}$. Then each $\beta_{i}$ is also a root of $x^{2 n-1}-1$. Suppose $\beta_{i}^{p^{l}-1}=1$ for all $1 \leqslant i \leqslant 2 n-2$. Then each $\beta_{i}$ is a root of $x^{p^{l}-1}-1$. This implies that $x^{p^{l}-1}-1=\left(x^{2 n-1}-1\right) w(x)$ for some $w(x) \in \mathbb{Z}_{p}[x]$. By Lemma 11, $p^{l} \equiv 1 \bmod (2 n-1)$, a contradiction. So, $\beta_{i_{0}}^{p^{l}-1} \neq 1$ for some $1 \leqslant i_{0} \leqslant 2 n-2$. Let $\beta_{i_{0}}^{p^{l}-1}$ be a root of $q_{j_{0}}(x)$. Let $E$ be a field extension of $\mathbb{Z}_{p}$ containing $\beta_{i_{0}}^{p^{l}-1}$. By Lemma 8 , the Galois group $G\left(E / \mathbb{Z}_{p}\right)$ is a cyclic group with generator $\eta$. Note that $\eta^{l}\left(\beta_{i_{0}}^{p^{l}-1}\right)=\beta_{i_{0}}^{\left(p^{l}-1\right) p^{l}}=\beta_{i_{0}}^{\left(p^{2 l}-1\right)+1-p^{l}}=\beta_{i_{0}}^{-\left(p^{l}-1\right)}$ where the last equality follows from $\beta_{i_{0}}^{2 n-1}=1$ and $p^{2 l}-1 \equiv 0 \bmod 2 n-1$. So, $\beta_{i_{0}}^{-\left(p^{l}-1\right)}$ is a root of $q_{j_{0}}(x)$. On the other hand, $\beta_{i_{0}}^{-\left(p^{l}-1\right)}$ is also a root of the monic irreducible polynomial $\frac{q_{j_{0}}^{*}(x)}{q_{j_{0}}(0)}$ (Lemma 4 ). This means $q_{j_{0}}(x)=\frac{q_{j_{0}}^{*}(x)}{q_{j_{0}}(0)}$. By Lemma $3, \frac{q_{j_{0}}^{*}(x)}{q_{j_{0}}(0)}$ is an irreducible factor of $f_{\mathbf{a}}^{*}(x)$. Therefore, $\beta_{i_{0}}^{-\left(p^{l}-1\right)}$ a root of $\sum_{i=0}^{2 n-2} x^{i}$ of multiplicity at least 2 , a contradiction. Hence, the order of $p$ is odd in $U_{2 n-1}$. This proves part (a) of the theorem.

By part (a) of the theorem and Lemma 10, the degree of $q_{i}(x)$ is odd and $q_{i}(0)=-1$ for $1 \leqslant i \leqslant m$. Then by Lemma 3 ,

$$
\begin{aligned}
f_{\mathbf{a}}^{*}(x) & =a_{n-1} q_{1}(0) q_{2}(0) \ldots q_{m}(0)\left(\frac{q_{1}^{*}(x)}{q_{1}(0)}\right)\left(\frac{q_{2}^{*}(x)}{q_{2}(0)}\right) \ldots\left(\frac{q_{m}^{*}(x)}{q_{m}(0)}\right) \\
& =a_{n-1}(-1)^{m}\left(-q_{1}^{*}(x)\right)\left(-q_{2}^{*}(x)\right) \ldots\left(-q_{m}^{*}(x)\right)
\end{aligned}
$$

where each $-q_{i}^{*}(x)$ is a monic irreducible polynomial (Lemma 4). Therefore $(-1)^{m} k \equiv$ $a_{n-1}^{2} \bmod p$. Let $e_{i}$ be the degree of $q_{i}(x)$. The degree of $f_{\mathbf{a}}(x) f_{\mathbf{a}}^{*}(x)$ is $2 \sum_{i=1}^{m} e_{i}$. So, $2 \sum_{i=1}^{m} e_{i}=2 n-2$, i.e., $\sum_{i=1}^{m} e_{i}=n-1$. Since each $e_{i}$ is odd, we have $m \equiv \sum_{i=1}^{m} e_{i} \equiv n-1$ $\bmod 2$. Hence, $(-1)^{m}=(-1)^{n-1}$ and part (b) of the theorem follows.

$(\Leftarrow)$ Suppose (a) and (b) hold. Note that $\left(\sum_{i=0}^{2 n-2} x^{i}\right)^{*}=\sum_{i=0}^{2 n-2} x^{i}$. So, if $\beta$ is a root of $\sum_{i=0}^{2 n-2} x^{i}$, then $\beta^{-1}$ is also a root of $\sum_{i=0}^{2 n-2} x^{i}$. This means that if $h(x)$ is a monic irreducible polynomial appearing in the factorization of $\sum_{i=0}^{2 n-2} x^{i}$, then by Lemma 4 and 10, $-h^{*}(x)$ is also a monic irreducible polynomial appearing in the factorization of $\sum_{i=0}^{2 n-2} x^{i}$. Furthermore, the degree of $h(x)$ and $-h^{*}(x)$ are odd and $-h^{*}(x) \neq h(x)$. So, we may write

$$
\sum_{i=0}^{2 n-2} x^{i}=h_{1}(x) h_{2}(x) \ldots h_{l}(x)\left(-h_{1}^{*}(x)\right)\left(-h_{2}^{*}(x)\right) \ldots\left(-h_{l}^{*}(x)\right)
$$


If $f_{i}$ is the degree of $h_{i}$, then $l \equiv \sum_{i=1}^{l} f_{i} \equiv n-1 \bmod 2$. Therefore $(-1)^{l}=(-1)^{n-1}$. Since $(-1)^{n-1} k$ is a quadratic residue modulo $p$, there exists an $a_{n-1} \in \mathbb{Z}_{p} \backslash\{0\}$ with $a_{n-1}^{2} \equiv(-1)^{n-1} k$. Now, there exists a unique $\mathbf{b}=\left(b_{0}, b_{1}, \ldots, b_{n-2}, 1\right)$ with $f_{\mathbf{b}}(x)=$ $h_{1}(x) h_{2}(x) \ldots h_{l}(x)$. Let $\mathbf{a}=a_{n-1} \mathbf{b}=\left(a_{n-1} b_{0}, a_{n-1} b_{1}, \ldots, a_{n-1} b_{n-2}, a_{n-1}\right)$. Then $f_{\mathbf{a}}(x)=$ $a_{n-1} h_{1}(x) h_{2}(x) \ldots h_{l}(x)$ and by Lemma $3, f_{\mathbf{a}}^{*}(x)=a_{n-1} h_{1}^{*}(x) h_{2}^{*}(x) \ldots h_{l}^{*}(x)$. Therefore,

$$
\begin{aligned}
f_{\mathbf{a}}(x) f_{\mathbf{a}}^{*}(x) & =a_{n-1}^{2} h_{1}(x) h_{2}(x) \ldots h_{l}(x) h_{1}^{*}(x) h_{2}^{*}(x) \ldots h_{l}^{*}(x) \\
& =a_{n-1}^{2}(-1)^{l} h_{1}(x) h_{2}(x) \ldots h_{l}(x)\left(-h_{1}^{*}(x)\right)\left(-h_{2}^{*}(x)\right) \ldots\left(-h_{l}^{*}(x)\right) \\
& =k \sum_{i=0}^{2 n-2} x^{i} .
\end{aligned}
$$

Hence, $\mathbf{a}$ is a $(\bar{k}, p)$-sequence (Lemma 5$)$.

Finally, note that $a_{n-1}$ and $-a_{n-1}$ are roots of $x^{2}-(-1)^{n-1} k$. We may choose $q_{i}=h_{i}(x)$ or $-h_{i}^{*}(x)$ for $1 \leqslant i \leqslant l$ and set $g_{\mathbf{c}}(x)= \pm a_{n-1} q_{1}(x) q_{2}(x) \ldots q_{l}(x)$. Then $\mathbf{c}$ is also a $(\bar{k}, p)$ sequence. So, if such a sequence exists, there are exactly $2^{l}$ of them if $p=2$ and $2^{l+1}$ if $p$ is odd. This completes the proof of the theorem.

Note that when $p=2$, Theorem 12 is the same as Theorem 2. So, Theorem 12 can be considered as a generalization of Theorem 2 .

Recall that $\mathbf{y}_{k}=\left(y_{0}, y_{1}, y_{2}, \ldots\right)$ with $y_{i}=(-1)^{i} k$. If $k \equiv 0 \bmod p$, then $\mathbf{y}_{k}=\overline{0}$. This case has been considered in Corollary 6 . So, we may assume $k \in \mathbb{Z}_{p} \backslash\{0\}$. If $p=2$, then $\mathbf{y}_{k}=\overline{1}$. This case has been considered in Theorem 2 and 12. So, we may assume that $p$ is an odd prime.

\section{Lemma 13.}

(a) Suppose $n$ is odd. Then $\mathbf{a}=\left(a_{0}, a_{1}, \ldots, a_{n-1}\right)$ is a $(\bar{k}, p)$-sequence if and only if $\mathbf{b}=\left(a_{0},-a_{1}, \ldots,(-1)^{n-1} a_{n-1}\right)$ is a $\left(\mathbf{y}_{k}, p\right)$-sequence.

(b) Suppose $n$ is even. Then $\mathbf{a}=\left(a_{0}, a_{1}, \ldots, a_{n-1}\right)$ is $a(\overline{-k}, p)$-sequence if and only if $\mathbf{b}=\left(a_{0},-a_{1}, \ldots,(-1)^{n-1} a_{n-1}\right)$ is a $\left(\mathbf{y}_{k}, p\right)$-sequence.

Proof. By Lemma 5, b is a $\left(\mathbf{y}_{k}, p\right)$-sequence if and only if

$$
\begin{aligned}
f_{\mathbf{b}}(x) f_{\mathbf{b}}^{*}(x) & =\sum_{i=0}^{n-1}(-1)^{n-1-i} k x^{i}+x^{n-1} \sum_{i=1}^{n-1}(-1)^{i} k x^{i} \\
& =(-1)^{n-1} k \sum_{i=0}^{2 n-2}(-1)^{i} x^{i} .
\end{aligned}
$$

Suppose $n$ is odd. Then $f_{\mathbf{b}}(x) f_{\mathbf{b}}^{*}(x)=k \sum_{i=0}^{2 n-2}(-1)^{i} x^{i}$. By Lemma 5 , a is a $(\bar{k}, p)$ sequence if and only if

$$
f_{\mathbf{a}}(x) f_{\mathbf{a}}^{*}(x)=k \sum_{i=0}^{2 n-2} x^{i}
$$


Hence, part (a) of the lemma follows by noting that $f_{\mathbf{b}}(x)=f_{\mathbf{a}}(-x)$ and $f_{\mathbf{a}}(x)=f_{\mathbf{b}}(-x)$.

Suppose $n$ is even. Then $f_{\mathbf{b}}(x) f_{\mathbf{b}}^{*}(x)=-k \sum_{i=0}^{2 n-2}(-1)^{i} x^{i}$. By Lemma 5 , a is a $(\overline{-k}, p)-$ sequence if and only if

$$
f_{\mathbf{a}}(x) f_{\mathbf{a}}^{*}(x)=-k \sum_{i=0}^{2 n-2} x^{i} .
$$

Hence, part (b) of the lemma follows by noting that $f_{\mathbf{b}}(x)=f_{\mathbf{a}}(-x)$ and $f_{\mathbf{a}}(x)=f_{\mathbf{b}}(-x)$.

Theorem 14. Let $p$ be an odd prime, $k \in \mathbb{Z}_{p} \backslash\{0\}$ and $\operatorname{gcd}(p, 2 n-1)=1=\operatorname{gcd}(p-$ $1,2 n-1) . A\left(\mathbf{y}_{k}, p\right)$-sequence of length $n>1$ exists if and only if

(a) the order of $p$ is odd in $U_{2 n-1}$,

(b) $k$ is a quadratic residue modulo $p$.

Furthermore, if such a sequence exists, then there are exactly $2^{l+1}$ of them, where $2 l$ is the number of irreducible factors of $\sum_{i=0}^{2 n-2} x^{i}$.

Proof. Suppose $n$ is odd. By part (a) of Lemma 13, there is a $\left(\mathbf{y}_{k}, p\right)$-sequence of length $n>1$ if and only if there is a $(\bar{k}, p)$-sequence of length $n>1$. Hence, Theorem 14 follows from Theorem 12 by noting that $(-1)^{n-1} k=k$.

Suppose $n$ is even. By part (a) of Lemma 13, there is a $\left(\mathbf{y}_{k}, p\right)$-sequence of length $n>1$ if and only if there is a $(\overline{-k}, p)$-sequence of length $n>1$. Hence, Theorem 14 follows from Theorem 12 by noting that $(-1)^{n-1}(-k)=k$.

Corollary 15. Let $p$ be a prime, $k \in \mathbb{Z}_{p} \backslash\{0\}$ and $\operatorname{gcd}(p, 2 n-1)=1=\operatorname{gcd}(p-1,2 n-1)$. If there is a $(\bar{k}, p)$-sequence or a $\left(\mathbf{y}_{k}, p\right)$-sequence of length $n>1$, then $p$ is a quadratic residue modulo $2 n-1$.

Proof. By Theorem 12 or 14 , the order of $p$ is odd in $U_{2 n-1}$. Let $2 e+1$ be the order of $p$. Then $\left(p^{e+1}\right)^{2} \equiv p^{2 e+2} \equiv p \bmod (2 n-1)$. Thus, $p$ is a quadratic residue modulo $2 n-1$.

Part (a) of the following Corollary was proved by Inglis and Wiseman [2, Proposition 1]. It was asked by Alles [1, Problem (1)].

Corollary 16. Let $p$ be a prime, $k \in \mathbb{Z}_{p} \backslash\{0\}$ and $\operatorname{gcd}(p, 2 n-1)=1=\operatorname{gcd}(p-1,2 n-1)$. Suppose there is a $(\bar{k}, p)$-sequence or a $\left(\mathbf{y}_{k}, p\right)$-sequence of length $n>1$. Then

(a) $n \equiv 0$ or $1 \bmod 4$, if $p=2$;

(b) $n \equiv 0$ or $1 \bmod 6$, if $p=3$;

(c) $n \equiv 0$ or $1 \bmod 5$, if $p=5$;

(d) $n \equiv 0,1,10,13,15,16,19,24,27,28,30,33 \bmod 42$, if $p=7$. 
Proof. (a) By Corollary 15, 2 is a quadratic residue modulo $2 n-1$. Let $q$ be a prime appearing in the factorization of $2 n-1$. Then $q$ is odd and 2 is a quadratic residue modulo q. Therefore $q \equiv 1$ or $7 \bmod 8$. This implies that $2 n-1 \equiv 1$ or $7 \bmod 8$. Thus, $n \equiv 1$ or $0 \bmod 4$.

(b) Since $\operatorname{gcd}(3,2 n-1)=1$, we require $2 n-1 \equiv 1$ or $2 \bmod 3$, that is $n \equiv 1$ or 0 mod 3. By Corollary 15, 3 is a quadratic residue modulo $2 n-1$. If $q$ is a prime appearing in the factorization of $2 n-1$, then $q$ is odd and 3 is a quadratic residue modulo $q$. By the Quadratic Reciprocity Law, $q \equiv \pm 1 \bmod 12$. This implies that $2 n-1 \equiv 1$ or 11 $\bmod 12$. Thus, $n \equiv 1$ or $0 \bmod 6$.

(c) Since $\operatorname{gcd}(5,2 n-1)=1$, we require $2 n-1 \equiv 1,2,3$, or $4 \bmod 5$, that is $n \equiv 1,4,2$, or $0 \bmod 5$. As in part (b), if $q$ is a prime appearing in the factorization of $2 n-1$, then 5 is a quadratic residue modulo $q$. By the Quadratic Reciprocity Law, $q \equiv \pm 1 \bmod 5$. This implies that $2 n-1 \equiv 1$ or $4 \bmod 5$. Thus, $n \equiv 1$ or $0 \bmod 5$.

(d) Since $\operatorname{gcd}(7,2 n-1)=1$ and $\operatorname{gcd}(6,2 n-1)=1, n \not \equiv 4 \bmod 7$ and $n \not \equiv 2 \bmod 3$. As before, if $q$ is a prime appearing in the factorization of $2 n-1$, then 7 is a quadratic residue modulo $q$. By the Quadratic Reciprocity Law, $q \equiv \pm 1, \pm 3$, or $\pm 9 \bmod 28$. This implies that $2 n-1 \equiv \pm 1, \pm 3$, or $\pm 9 \bmod 28$. Thus, $n \equiv 0,1,2,5,10$, or $13 \bmod 14$. Since $n \not \equiv 2 \bmod 3$ and $\operatorname{gcd}(3,14)=1$, we must have $n \equiv 0,28,1,15,16,30,19,33,10$, 24,13 or $27 \bmod 42$.

\section{Acknowledgments}

We would like to thank the anonymous referee for the comments that helped us make several improvements to this paper. This project was supported by the Frontier Science Research Cluster, University of Malaya (RG367-15AFR).

\section{References}

[1] P. Alles, On a conjecture of J. Pelikán, J. Combin. Theory Ser. A 60 (1992), 312-313.

[2] N.F.J. Inglis and J.D.A. Wiseman, Very odd sequences, J. Combin. Theory Ser. A 71 (1995), 89-96.

[3] N. Jacobson, Basic Algebra I, second edition, W.H. Freeman and Company, U.S.A., 1985.

[4] F.J. MacWilliams and A.M. Odlyzko, Pelikan's conjecture and cyclotomic cosets, J. Combin. Theory Ser. A 22 (1977), 110-114.

[5] P. Moree and P. Solé, Around Pelikán's conjecture on very odd sequences, Manuscripta Math. 117, (2005), 219-238.

[6] J. Pelikán, Contribution to "Problems", Colloq. Math. Soc. János Bolyai 10 p. 1549, North-Holland, Amsterdam, 1975. 